\title{
Factors that influence hospital inpatients' exercise constraints
}

\author{
Sang-Wan Jeon, Eun-Surk Yi* \\ Exercise Rehabilitation Convergence Institute, Gachon University, Incheon, Korea
}

The purpose of this study is to present ways to provide more precise patient-oriented services by presenting implications and promoting a better understanding of exercise constraints. This study used questionnaire survey as measurement tool for 196 hospital inpatients in 5 general hospitals in the metropolitan area (Seoul, Gyeonggi, and Incheon). The collected data were analyzed using frequency analysis, exploratory factor analysis, reliability analysis, correlation analysis, and hierarchical regression analysis using IBM SPSS Statistics ver. 23.0. The following results were obtained. First, physical activity level and health condition on their physical constraints showed that their health condition factors such as pain/discomfort and anxiety/depression had greater effects on physical constraints than other factors. Second, physical activity level, and health condition on their psychological constraints showed that health condition factors had major effects on patients' psychological constraints. Thirdly, physical activity level, and health condition on their sociocultural constraints found that anxiety/depression appeared as the key influence factor in physical and psychological constraints.

Keywords: Inpatients, Exercise, Constraints, Empirical analysis, Hierarchical regression analysis, Hospital

\section{INTRODUCTION}

According to a report by Statics Korea, Korea has already become an aging society with the population over 65 years of age already accounting for $11.3 \%$ of total population as of 2010 , and based on this trend, it is expected to become an aged society by 2019 and a super-aged society by 2026 (Statics Korea, 2016). This phenomenon will increase the use of medical facilities such as hospitals by seniors, who face more health issues than other generations. In particular, the increased number of seniors leads to an increased number of different adult diseases and surgical patients. The medical costs of seniors over 65 years of age account for $35.5 \%$ of the costs of all patients, the increase in elderly patients over 75 years of age account for $66 \%$ of the overall patient cost increase. The increasing number of seniors will result in a rapid increase in the number of patients visiting hospitals, which may become a social and financial burden for both the individual and the nation. Therefore, general efforts by society to encourage a return to daily life through fast recovery and minimize the nation's social costs are needed.

Generally, physical activities are method of disease control and treatment for patients (Boujibar et al., 2018). In particular, exercise has positive effects on physical and mental health and is essential for patients who feel their quality of life has deteriorated due to physical, psychological, emotional, and financial hardships. However, most inpatients struggle to participate in recovery exercises due to physical limitations such as deteriorated stamina and muscular strength after different treatments as well as emotional limitations, such as depression, fear of death, and stress (Lewis, 1982). In addition, it is quite difficult to maintain physical activity during hospitalization Although physical activity is considered as an important factor in health recovery, there have been few systematic studies of physical activity of inpatients to prepare strategically for an aging society.

Therefore, this study conducted an empirical analysis of physical activity and factors that are related to insufficient inpatient

\footnotetext{
${ }^{*}$ Corresponding author: Eun-Surk Yi (iD https://orcid.org/0000-0002-9370-5231 Department of Exercise Rehabilitation \& Welfare, Gachon University, 191 Hombakmoe-ro, Yeonsu-gu, Incheon 21936, Korea Tel: +82-32-820-4442, Fax: +82-32-820-4449, E-mail: yies@gachon.ac.kr Received: May 26, 2018 / Accepted: June 21, 2018
}

This is an Open Access article distributed under the terms of the Creative Commons Attribution Non-Commercial License (http://creativecommons.org/licenses/by-nc/4.0/) which permits unrestricted non-commercial use, distribution, and reproduction in any medium, provided the original work is properly cited. 
physical activity based on a survey of adult patients hospitalized in 5 general hospitals in the metropolitan area (Seoul, Gyeonggi, and Incheon). It aims to present ways to provide more precise patient-oriented services by presenting implications and promoting a better understanding of exercise constraints.

\section{MATERIALS AND METHODS}

\section{Subjects}

Subjects were adult male and female patients over 20 years of age admitted to 5 general hospitals in the metropolitan area (Seoul, Gyeonggi, and Incheon). Written consent was obtained from 250 admitted patients selected through purposive sampling, and a survey was conducted to collect data. The survey period was from April to June 2017, and data from 196 subjects was used for the final analysis with data from 54 subjects who submitted unreliable responses excluded. The general characteristics of study subjects were as seen in Table 1.

Tools

Data was collected via a survey containing questions about sex,

Table 1. Demographic characteristics $(n=196)$

\begin{tabular}{lc}
\hline Variable & No. of cases (\%) \\
\hline Sex & \\
Male & $97(49.5)$ \\
Female & $99(50.5)$ \\
Age (yr) & \\
Below 40 & $55(28.1)$ \\
40 s & $24(12.2)$ \\
50 s & $53(27.0)$ \\
60 s & $37(18.9)$ \\
Over 70 & $27(13.7)$ \\
Education & \\
Middle school or below & $46(23.5)$ \\
High school & $75(38.3)$ \\
University & $63(32.1)$ \\
Higher than bachelor's degree & $12(6.1)$ \\
Income (KRW) & \\
Below 2 million & $85(43.4)$ \\
2 million-4 million & $55(28.1)$ \\
4 million-6 million & $37(18.9)$ \\
Over 6 million & $19(9.6)$ \\
Marital status & \\
Married & $140(71.4)$ \\
Single & $44(22.4)$ \\
Other & $12(6.2)$ \\
\hline
\end{tabular}

KRW, Korean won. age, educational background, income, and marital status to ascertain the general characteristics of subjects. The survey of personal exercise constraint factors used in a study by Zhu et al. (2001) was revised for this study, with 13 questions asked. There were 2 questions about inpatients' health factors inquiring about the history of surgery and exercise experience prior to hospitalization. Inpatients' physical activity level was measured via questions regarding 'time spent seated,' 'life maintenance time' such as eating, cleaning up, and dressing, and 'intentional moving time' related to physical activities such as walking and exercising. There were also 5 questions about health status from EuroQoL-5 Dimensions-5 Levels (EQ-5D-5L), developed by Herdman et al. (2011) to improve sensitivity and the ceiling effect of existing EQ-5D$3 \mathrm{~L}$, including questions about mobility, self-care, usual activities, pain/discomfort, and anxiety/depression.

\section{Survey reliability and validity}

The data collected in this study was tested for validity using confirmatory factor analysis. Principal component analysis was used to extract components, and varimax was adopted to simplify factor loadings. The Kaiser-Meyer-Olkin (KMO) Test was used as the reference in selecting variables for factor analysis. Bartlett sphericity test value $\left(\chi^{2}\right)$ was used to show the suitability of factor analysis use and common factors, and question selection accepted factors with 1.0 or higher eigenvalue.

In exploratory factor analysis, personal exercise constraints included 3 questions regarding physical constraints, 4 regarding psychological constraints, and 4 regarding sociocultural constraints. Specifically, personal exercise constraints resulted in $\mathrm{KMO}=0.818$, $\chi^{2}=1,054.425$, eigenvalue of physical constraints was 3.120 (variance \%: 28.362), eigenvalue of sociocultural constraints was 2.563 (variance \%: 23.303), and eigenvalue of physical constraints was 1.868 (variance \%: 16.980). All factor loadings were above 0.05 , and Cronbach a coefficient was used to verify the reliability of each variable. As a result, physician constraints were determined to be 0.676 , psychological constraints 0.676 , and sociocultural constraints 0.807 .

\section{Data processing}

The purpose of this study was to examine the effects of hospital inpatients' health factors, physical activity level, and health condition on their personal exercise constraints. For this, the researcher and assistant researcher visited hospitals and explained the purpose and goals of the survey after obtaining the hospital's cooperation. Patients completed the survey by self-reporting. Surveys with low 
reliability, double entries, and no entries were excluded from the analysis, and the remaining data was individually coded and used for frequency analysis, exploratory factor analysis, reliability analysis, correlation analysis, and hierarchical regression analysis using IBM SPSS Statistics ver. 23.0 (IBM Co., Armonk, NY, USA).

\section{RESULTS}

\section{Correlation among variables}

Prior to verifying the relationship between variables set in this study, a correlation analysis was performed to examine the correlation among the subvariables and their multicollinearity. These variables showed a correlation of $(P<0.05)$ as seen in Table 2 . The correlation coefficient among subvariables were below 0.80 , indicating no issues with multicollinearity. Therefore, each variable was verified to be independent. As seen in Table 2, all factors excluding 'sedentary lifestyle' showed some positive $(+)$ and negative (-) correlations.

\section{Effects of hospital inpatients' physical factors, physical activity level, and health condition on their personal exercise constraints}

Residual analysis using the regression model was performed prior to hierarchical regression analysis for the effects of inpatients'

Table 2. Correlation analysis

\begin{tabular}{|c|c|c|c|c|c|c|c|c|c|c|c|c|c|}
\hline & a & $b$ & c & $d$ & e & $f$ & $g$ & $\mathrm{~h}$ & i & j & k & 1 & $\mathrm{~m}$ \\
\hline$a$ & 1 & & & & & & & & & & & & \\
\hline$b$ & 0.044 & 1 & & & & & & & & & & & \\
\hline c & -0.022 & -0.016 & 1 & & & & & & & & & & \\
\hline$d$ & -0.099 & 0.109 & 0.018 & 1 & & & & & & & & & \\
\hline e & -0.089 & -0.067 & 0.007 & $0.271^{* *}$ & 1 & & & & & & & & \\
\hline$f$ & $0.347^{* *}$ & -0.046 & 0.023 & $-0.129^{*}$ & -0.064 & 1 & & & & & & & \\
\hline g & $0.260^{* *}$ & -0.006 & -0.067 & -0.018 & -0.031 & $0.679 * *$ & 1 & & & & & & \\
\hline h & $0.336^{* *}$ & 0.061 & 0.027 & -0.104 & $-0.163^{*}$ & $0.739 * *$ & $0.782^{* *}$ & 1 & & & & & \\
\hline i & $0.279^{* *}$ & 0.056 & 0.081 & -0.032 & -0.094 & $0.514^{* *}$ & $0.555^{* *}$ & $0.726^{* *}$ & 1 & & & & \\
\hline j & $0.162^{* *}$ & 0.001 & -0.094 & 0.044 & -0.066 & $0.339 * *$ & $0.511^{* *}$ & $0.415^{* *}$ & $0.383^{* *}$ & 1 & & & \\
\hline k & $0.185^{* *}$ & $-0.123^{*}$ & 0.060 & 0.048 & 0.076 & $0.350^{* *}$ & $0.399 * *$ & $0.422^{* *}$ & $0.447^{* *}$ & $0.395^{* *}$ & 1 & & \\
\hline I & $-0.124^{*}$ & $-0.349^{* *}$ & -0.088 & 0.083 & $0.233^{* *}$ & -0.029 & 0.099 & -0.024 & -0.061 & $0.214^{* *}$ & $0.319^{* *}$ & 1 & \\
\hline $\mathrm{m}$ & 0.041 & -0.112 & -0.074 & 0.113 & 0.116 & -0.094 & -0.088 & $-0.119^{*}$ & -0.089 & $0.166^{* *}$ & $0.151^{* *}$ & $0.426^{* *}$ & 1 \\
\hline
\end{tabular}

a, history of surgery; b, exercise experience; c, hours seated; $d$, daily routine time; e, active time; $f$, physical ability; $g$, self-management; $h$, daily activities; i, pain/discomfort; $j$, anxiety/depression; $k$, physical constraints; I, psychological constraints; $m$, sociocultural constraints.

${ }^{*} P<0.05$. ${ }^{* *} P<0.01$.

Table 3. Effects of health factors, physical activity level, and health condition on physical constraints

\begin{tabular}{|c|c|c|c|c|c|c|c|c|c|}
\hline \multirow{2}{*}{ Variable } & \multicolumn{3}{|c|}{ Model 1} & \multicolumn{3}{|c|}{ Model 2} & \multicolumn{3}{|c|}{ Model 3} \\
\hline & SE & $\beta$ & $t$ & SE & $\beta$ & $t$ & SE & $\beta$ & $t$ \\
\hline History of surgery & 0.118 & 0.191 & $2.717^{* *}$ & 0.119 & 0.204 & $2.891^{* *}$ & 0.111 & 0.044 & 0.670 \\
\hline Exercise experience & 0.113 & -0.132 & -1.877 & 0.115 & -0.134 & -1.884 & 0.101 & -0.142 & $-2.281^{*}$ \\
\hline Hours seated & - & - & - & 0.060 & 0.060 & 0.859 & 0.053 & 0.056 & 0.901 \\
\hline Daily routine hours & - & - & - & 0.059 & 0.063 & 0.861 & 0.052 & 0.050 & 0.768 \\
\hline Active hours & - & - & - & 0.046 & 0.068 & 0.923 & 0.041 & 0.118 & 1.810 \\
\hline Physical ability & - & - & - & - & - & - & 0.061 & 0.027 & 0.281 \\
\hline Self-management & - & - & - & - & - & - & 0.065 & 0.023 & 0.207 \\
\hline Daily activity & - & - & - & - & - & - & 0.087 & 0.129 & 0.961 \\
\hline Pain/discomfort & - & - & - & - & - & - & 0.062 & 0.242 & $2.679 * *$ \\
\hline Anxiety/depression & - & - & - & - & - & - & 0.053 & 0.232 & $3.198^{* *}$ \\
\hline Statistics & \multicolumn{3}{|c|}{$F=5.238, R^{2}=0.042$} & \multicolumn{3}{|c|}{$F=2.688, R^{2}=0.041$} & \multicolumn{3}{|c|}{$F=8.437, R^{2}=0.276$} \\
\hline
\end{tabular}

SE, standard error

${ }^{*} P<0.05 .{ }^{*} P<0.01$. 
physical factors, physical activity level, and health condition on their personal exercise constraints, and no outliers were found. Furthermore, there was no deviation from normality, homoscedasticity, and linearity. As seen in Tables 3-5, $F$ values were statistically significant, and the regression model had a linear relation.

\section{Effects of hospital inpatients' health factors, physical activity level, and health condition on their physical constraints}

The results of hierarchical regression analysis to explain the effects of hospital inpatients' health factors, physical activity level, and health condition on their physical constraints were as seen in Table 3. In models 1 and 2, inpatients' history of surgery had a statistically positive (+) effect on physical constraints. In contrast, in model 3, the history of surgery was eliminated from the significance level, but exercise experience factor showed a statistically negative (-) effect on physical constraints. Moreover, pain/discomfort and anxiety/depression had statistically positive (+) effects on physical constraints. Finally, the order of influence based on the comparison of standardized coefficients and absolute values was found to be pain/discomfort, anxiety/depression, and daily activities, and the explanation power of health factors, physical activity level, and health condition on physical constraints was $27.6 \%$.

\section{Effects of hospital inpatients' health factors, physical activity level, and health condition on their psychological constraints}

The result of hierarchical regression analysis to explain the hos-

Table 4. Effects of health factors, physical activity level, and health condition on psychological constraints

\begin{tabular}{|c|c|c|c|c|c|c|c|c|c|}
\hline \multirow{2}{*}{ Variable } & \multicolumn{3}{|c|}{ Model 1} & \multicolumn{3}{|c|}{ Model 2} & \multicolumn{3}{|c|}{ Model 3} \\
\hline & SE & $\beta$ & $t$ & SE & $\beta$ & $t$ & SE & $\beta$ & $t$ \\
\hline History of surgery & 0.110 & -0.109 & -1.624 & 0.108 & -0.088 & -1.339 & 0.113 & -0.099 & -1.441 \\
\hline Exercise experience & 0.106 & -0.345 & $-5.140 * * *$ & 0.104 & -0.342 & $-5.158^{* * *}$ & 0.102 & -0.340 & $-5.246^{* * *}$ \\
\hline Sitting hours & - & - & - & 0.054 & -0.098 & -1.339 & 0.054 & -0.056 & -0.873 \\
\hline Daily life maintenance hours & - & - & - & 0.054 & 0.063 & 0.914 & 0.053 & 0.033 & 0.491 \\
\hline Intentional moving hours & - & - & - & 0.042 & 0.186 & $2.717^{* *}$ & 0.041 & 0.205 & $3.030^{* *}$ \\
\hline Physical ability & - & - & - & - & - & - & 0.061 & -0.139 & -1.399 \\
\hline Self-management & - & - & - & - & - & - & 0.066 & 0.099 & 0.872 \\
\hline Daily activity & - & - & - & - & - & - & 0.088 & 0.085 & 0.611 \\
\hline Pain/discomfort & - & - & - & - & - & - & 0.063 & -0.129 & -1.374 \\
\hline Anxiety/depression & - & - & - & - & - & - & 0.054 & 0.248 & $3.293^{* *}$ \\
\hline Statistics & \multicolumn{3}{|c|}{$F=14.923, R^{2}=0.125$} & \multicolumn{3}{|c|}{$F=8.766, R^{2}=0.166$} & \multicolumn{3}{|c|}{$F=6.470, R^{2}=0.219$} \\
\hline
\end{tabular}

SE, standard error.

${ }^{* *} P<0.01$. ${ }^{* *} P<0.001$.

Table 5. Effects of health factors, physical activity level, and health condition on sociocultural constraints

\begin{tabular}{|c|c|c|c|c|c|c|c|c|c|}
\hline \multirow{2}{*}{ Variable } & \multicolumn{3}{|c|}{ Model 1} & \multicolumn{3}{|c|}{ Model 2} & \multicolumn{3}{|c|}{ Model 3} \\
\hline & SE & $\beta$ & $t$ & SE & $\beta$ & $t$ & SE & $\beta$ & $t$ \\
\hline History of surgery & 0.132 & 0.046 & 0.643 & 0.132 & 0.063 & 0.883 & 0.138 & 0.102 & 1.369 \\
\hline Exercise experience & 0.126 & -0.114 & -1.597 & 0.127 & -0.123 & -1.709 & 0.125 & -0.119 & -1.686 \\
\hline Hours seated & - & - & - & 0.066 & -0.077 & -1.709 & 0.066 & -0.053 & -0.754 \\
\hline Daily routine hours & - & - & - & 0.066 & 0.111 & 1.490 & 0.065 & 0.085 & 1.161 \\
\hline Active hours & - & - & - & 0.051 & 0.084 & 1.126 & 0.050 & 0.096 & 1.302 \\
\hline Physical ability & - & - & - & - & - & - & 0.075 & -0.046 & -0.421 \\
\hline Self-management & - & - & - & - & - & - & 0.081 & -0.182 & -1.471 \\
\hline Daily activity & - & - & - & - & - & - & 0.108 & -0.009 & -0.060 \\
\hline Pain/discomfort & - & - & - & - & - & - & 0.077 & -0.074 & -0.724 \\
\hline Anxiety/depression & - & - & - & - & - & - & 0.066 & 0.289 & $3.518^{* *}$ \\
\hline Statistics & \multicolumn{3}{|c|}{$F=1.438, R^{2}=0.004$} & \multicolumn{3}{|c|}{$F=1.758, R^{2}=0.019$} & \multicolumn{3}{|c|}{$F=2.482, R^{2}=0.071$} \\
\hline
\end{tabular}

SE, standard error.

${ }^{* *} P<0.01$ 
pital inpatients' health factors, physical activity level, and health condition on their psychological constraints was as seen in Table 4. In models 1, 2, 3, inpatients' exercise experience had a statistically negative (-) effect on psychological constraints, and in models 2 and 3 , active hours had statistically positive $(+)$ effects on inpatients' psychological constraints. In model 3 , anxiety/depression had a statistically positive $(+)$ effect on psychological constraints. Finally, the order of influence based on the comparison of standardized coefficients and absolute values was anxiety/depression, exercise ability, and pain/discomfort, and the explanation power of health factors, physical activity level, and health condition on psychological constraints was $21.9 \%$.

\section{Effects of hospital inpatients' health factors, physical activity level, and health condition on their sociocultural constraints}

The result of hierarchical regression analysis to explain the hospital inpatients' health factors, physical activity level, and health condition on their sociocultural constraints was as seen in Table 5 . In models 1 and 2, inpatients' health factors and physical activity level had no statistically significant effect on sociocultural constraints, whereas in model 3 , only anxiety/depression showed a statistically positive $(+)$ effect on physical constraints. Finally, the order of influence based on the comparison of standardized coefficients and absolute values was anxiety/depression, self-management, and pain/discomfort, and the explanation power of health factors, physical activity level and health condition on sociocultural constraints was $7.1 \%$.

\section{DISCUSSION}

The purpose of this study was to empirically examine the factors that affect hospital inpatients' personal exercise constraints and to analyze the effects of inpatients' health factors, physical activity level, and health condition on their personal exercise constraints; the following discussion can be made based on the data obtained from study results.

First, hierarchical regression analysis of the effects of hospital inpatients' health factors, physical activity level and health condition on their physical constraints showed that their health condition factors such as pain/discomfort and anxiety/depression had greater effects on physical constraints than other factors. Generally, patients tend to refrain from physical activities due to pain that arises from postoperative recovery and treatment, frustration that they cannot move freely during the treatment period, and fear of recurrence. With regard to this, a previous study also reported that negative emotions such as anxiety or depression after feeling greater pain than expected restrict physical activities (Wassink-Vossen et al., 2014). In particular, physical activities to promote individual recovery, unlike rehabilitation for therapeutic purposes, only aggravated a different kind of psychological anxiety due to lack of exercise techniques and information. Meanwhile, exercise experience prior to hospitalization had a negative effect on physical constraints. In other words, as many previous studies have reported that previous exercise experience is an important variable that significantly predicts exercise-related awareness, it can be predicted that previous exercise experience leads to action to feel less pain and overcome it, although physical pain and discomfort may accompany this (Conn, 1998a). Therefore, efforts by medical institutions to educate patients regarding the importance of exercise are needed to increase exercise behavior.

Second, hierarchical regression analysis on the effects of hospital inpatients' health factors, physical activity level, and health condition on their psychological constraints showed that health condition factors had major effects on patients' psychological constraints. In particular, anxiety surrounding the possibility of recovery, negative emotions about the situation that the patient is in, and pain/discomfort acted as constraints in deciding on and implementing exercise participation. This means that anxiety/depression and pain/discomfort act as serious psychological factors in participating in exercise, which is supported by the results of a previous study that found osteoarthritis patients tend to avoid exercise due to the fear that it worsens symptoms (Semble et al., 1990). Generally, perceived behavior-related factors such as benefit, self-efficacy, disability, and enjoyment significantly predicted exercise behavior in an empirical study (Conn, 1998b; Johnson and Heller, 1988). Perceived behavior-related factors may readily change inpatients' exercise constraining factors and may serve as the key in mediation. In addition, deterioration of motor ability appeared among physical constraint effect factors such as interest in exercise or fear of injury.

Thirdly, hierarchical regression analysis on the effects of hospital inpatients' health factors, physical activity level, and health condition on their sociocultural constraints found that anxiety/depression appeared as the key influence factor in physical and psychological constraints. Generally, it is not easy for inpatients to find inner peace and get back on life during treatment and recovery. These results from various factors such as recovery status or treatment stability, and with patients becoming mentally unstable, this negative emotional state may prevent them from accepting the help or exercise information needed for recovery. Furthermore, 
it is not easy for hospitalized patients to accept being diagnosed with disease and perform appropriate exercise at the same time. A relevant previous study reported that hemodialysis patients with terminal renal disease were affected in terms of physical constraints by social/environmental disability factors such as unwillingness to exercise, lack of time, and lack of exercise partner (Delgado and Johansen, 2012).

Therefore, considering that pain, anxiety, or depression arising from the treatment process affect patients' personal exercise constraints, programs and education that can manage such pain, anxiety, and depression need to be provided. Moreover, a medical service environment where admitted patients can voluntarily participate in exercise should be established.

This study would like to make the following proposals based on these results. This study focused on the individual aspect of inpatients' exercise constraints. Therefore, follow-up studies should categorize social and environmental characteristics as well as individual exercise constraints in more depth to analyze inpatients' exercise constraints from different angles. This is because the exercise constraints of hospital inpatients may vary due to personal or other environmental factors. Psychological constraints in particular were not thoroughly examined through in-depth patient interviews and observation. Therefore, follow-up studies are needed to categorize hospital inpatients' exercise constraint factors based on various inpatient groups to perform a qualitative study that can analyze why physical exercise is so difficult in hospital environments. This is because such exercise is significantly related to the improvement of services at medical institutions as well as patients' fast recovery.

\section{CONFLICT OF INTEREST}

No potential conflict of interest relevant to this article was reported.

\section{ACKNOWLEDGMENTS}

This work was supported by the Ministry of Education of the Republic of Korea and the National Research Foundation of Korea (NRF-2016S1A5B6913737).

\section{REFERENCES}

Boujibar F, Bonnevie T, Debeaumont D, Bubenheim M, Cuvellier A, Peillon C, Gravier FE, Baste JM. Impact of prehabilitation on morbidity and mortality after pulmonary lobectomy by minimally invasive surgery: a cohort study. J Thorac Dis 2018;10:2240-2248.

Conn VS. Older adults and exercise: path analysis of self-efficacy related constructs. Nurs Res 1998a;47:180-189.

Conn VS. Older women's beliefs about physical activity. Public Health Nurs 1998b;15:370-378.

Delgado C, Johansen KL. Barriers to exercise participation among dialysis patients. Nephrol Dial Transplant 2012;27:1152-1157.

Herdman M, Gudex C, Lloyd A, Janssen M, Kind P, Parkin D, Bonsel G, Badia X. Development and preliminary testing of the new five-level version of EQ-5D (EQ-5D-5L). Qual Life Res 2011;20:1727-1736.

Johnson NA, Heller RF. Prediction of patient nonadherence with homebased exercise for cardiac rehabilitation: the role of perceived barriers and perceived benefits. Prev Med 1998;27:56-64.

Lewis FM. Experienced personal control and quality of life in late-stage cancer patients. Nurs Res 1982;31:113-119.

Semble EL, Loeser RF, Wise CM. Therapeutic exercise for rheumatoid arthritis and osteoarthritis. Semin Arthritis Rheum 1990;20:32-40.

Statistics Korea. Future population estimation. Daejeon (Korea): Statistics Korea; 2016.

Wassink-Vossen S, Collard RM, Oude Voshaar RC, Comijs HC, de Vocht HM, Naarding P. Physical (in)activity and depression in older people. J Affect Disord 2014;161:65-72.

Zhu W, Timm G, Ainsworth B. Rasch calibration and optimal categorization of an instrument measuring women's exercise perseverance and barriers. Res Q Exerc Sport 2001;72:104-116. 